\title{
Transparent free relatives with who: Support for a unified analysis
}

\author{
Carson T. Schütze \& Richard Stockwell*
}

\begin{abstract}
This paper provides novel data, including from acceptability ratings, supporting a unified analysis of Transparent Free Relatives (TFRs) as variants of Standard Free Relatives (SFRs), rather than entirely different beasts. Two arguments are presented. First, who-TFRs exist, contrary to the view in the literature that TFRs can only be formed with what. Second, who-TFRs degrade following the same illunderstood pattern as who-SFRs. These outcomes cohere better with accounts of TFRs that treat them as similar to SFRs, versus accounts that treat them as virtually unrelated.
\end{abstract}

Keywords. standard free relatives; transparent free relatives; degradation of who free relatives; $w h$-words

1. Introduction. This paper provides novel data supporting a unified analysis of Transparent Free Relatives (TFRs) (1) and Standard Free Relatives (SFRs) (2). While the precise criteria for identifying TFRs are not entirely settled, it is widely agreed that one necessary property is that the base trace of the $w h$-word be in the subject position of a small clause (SC). Thus, (1) could be a TFR while (2) could not. (Further properties will be discussed shortly.)

(1) John saw [TFR what he believed to be [sc $t$ raccoons]] outside.

(2) John ate [SFR what Mary cooked $t]$.

TFRs are often analyzed as radically different from SFRs, which are generally treated like headed relative clauses up to Spec,CP. TFRs are commonly treated with the SC predicate (raccoons in (1)) as an 'internal head' that appears in two structurally distinct configurations: one reflecting its "downstairs" function (being predicated of (the trace of) what); the other reflecting its apparent "upstairs" function (object of $s a w$ ). This dual functionality is implemented either using multiple dominance, in what amounts to an amalgam structure (cf. van Riemsdijk 2006), or two copies, one of which is elided under identity, in what amounts to a parenthetical (Wilder 1999; cf. Schelfout et al. 2004). The latter approach is meant to suggest paraphrases like John saw raccoons outside (or at least, what he believed to be raccoons), though there is debate over whether this accurately reflects the meaning of (1). The main appeal of these non-unified approaches is that they can readily account for properties of TFRs that are not found in SFRs (see below).

By contrast, a unified account, of which various versions have been proposed by Grosu (2003, 2016), posits that TFRs wear their structure on their sleeve, like SFRs: internally, they too look like headed relatives (cf. John saw creatures which he believed to be raccoons outside) and thus do not attribute any dual status to the SC predicate. On this approach it takes more work to explain properties of TFRs that they do not share with SFRs.

\footnotetext{
* Our thanks to four anonymous reviewers; the audience at our poster at the LSA; and to Ivano Caponigro, Alex Grosu, Jesse Harris, Stefan Keine and Yael Sharvit. This research was supported by a UCLA Faculty Senate Grant to the first author. All the errors are ours. Authors: Carson T. Schütze, University of California, Los Angeles (cschutze@ucla.edu) \& Richard Stockwell, University of California, Los Angeles (rstockwell15@ucla.edu).
} 
2. Distinguishing TFRs from SFRs. There is debate over what the total set of properties unique to TFRs is (van Riemsdijk 2017), so we limit discussion to the three that have been most widely agreed upon.

First, while SFRs can be introduced by a wide range of $w h$-words, TFRs can only be introduced by what (3) (cf. Wilder 1999):

(3) John should have married [TFR what $/{ }^{*}$ who his mother would call [ $t$ a traditional woman]].

Second, TFRs can trigger plural verbal agreement, while SFRs cannot (4). The SFR in (b) disallows a plural matrix verb even in a context where we know that I see multiple things that scare me.

(4) a. what-TFR: [TFR What seem to be [sc $t$ raccoons]] are/*is eating our garbage.

b. what-SFR: $\quad[$ SFR What I see $t]$ scares/*scare me.

Third, TFRs can receive "indefinite" interpretations, while SFRs can only receive definite interpretations. This claim has been substantiated in two ways. First, TFRs can be used in contexts where a headed relative paraphrase with an indefinite article sounds felicitous while one with a definite article does not. For example, out of the blue (1) seems to mean John saw some creatures he thought were raccoons outside, not John saw the creatures he thought were raccoons outside. Second, a TFR can appear as the "associate" in an existential there sentence, while an SFR cannot (5):

(5) a. what-TFR: There was [TFR what he believed to be [sC $t$ a raccoon]] outside.

b. what-SFR: *There was [SFR what she cooked $t]$ on the table.

It should be acknowledged that the precise property excluded by the existential there frame is not strictly definiteness (cf. There was the most amazing documentary on TV last night), and thus, although there is little dispute that SFRs are always interpreted as definites, there is a subclass of SFRs that can appear in such sentences (Hinterwimmer 2008):

(6) There was [SFR what Mary likes to wear $t]$ in the closet.

In (6) the SFR is interpreted as 'the kind of thing that Mary likes to wear', which differs from 'the stuff Mary cooked' in not referring to an individual that must be assumed to be familiar in the context. Thus, in using existential sentences to diagnose TFRs it is important that the FR not receive a kind interpretation.

Our goal in the rest of the paper is to argue that there are FRs that display the characteristics of TFRs but that, contrary to the first claim above, are introduced by who. If so, that would reduce the number of properties that are special to TFRs, making it easier to assimilate them to SFRs.

3. Data collection. We provide new acceptability rating data to substantiate our findings. 60 native American English speakers were recruited on Amazon Mechanical Turk and asked to rate sentences on a 1 (lowest) - 7 (highest) Likert scale. Each subject rated a total of 50 sentences, about half of which were for an unrelated experiment. Examples are annotated with mean ratings throughout the paper. Given the exploratory nature of the data collection, statistical comparisons have been limited to $t$-tests and are intended as suggestive only. Ungrammatical (7) and grammatical (8) sentences not involving FRs received appropriately low and high ratings, respectively, and serve as baselines for comparison with later examples. 
(7) a. *Promise to wash, Neal did the car. $\quad 1.78$

b. *They consider of teacher a Chris geeky. $\quad 1.18$

(8) She was the winner of the grand prize. 6.98

4. TFRs can be introduced by who. Examples with the shape of TFRs but introduced by who are attested on the Web (9), and were highly rated by our subjects. Constructed examples (10) also received high ratings. Note that these examples naturally receive indefinite rather than definite paraphrases.

(9) a. I once saw [TFR who I thought was [sc $t$ Robert Redford]] at a Starbucks.

b. Have a look through the picture gallery and you'll see [TFR who is certain to be [sc $t$ one of our cutest fans]].

(10) a. After the collision, Rhonda was rescued by [TFR who she suspects was [sc $t$ a highway patrol officer]].

b. Although my eyesight is poor, I can see [TFR who I assume to be [sc $t$ a soldier]] in the distance.

Placing potential who-TFRs in the existential construction from (5) yields results that to our intuitions are comparable to those for similarly-constructed what-TFRs, and clearly better than SFRs introduced by who or what (11). We have not yet tested a full paradigm experimentally, and can report ratings only for the who-TFR. ${ }^{1}$

(11) a. what-TFR: There were [TFR what he believed to be [sc $t$ raccoons]] outside.

b. who-TFR: There are [TFR who the president believes to be [SC $t$ gang members]] in the caravan.

c. what-SFR: $\quad$ *There was [SFR what she cooked $t$ ] on the table.

d. who-SFR: $\quad$ *There was [SFR who Mary fired $t$ ] at the party.

The purported who-TFRs can also trigger plural agreement more readily than who-SFRs. Across similar pairs of who-FRs with plural agreement, the TFR (a) was rated significantly higher than the SFR (b) in both (12) $(p<.01)$ and (13) $(p<.02)$, despite the fact that the SFRs readily lend themselves to scenarios where their referent would be plural.
a. who-TFR: [TFR Who the FBI labelled [sc $t$ suspects]] have been released for lack of evidence.
b. who-SFR: [SFR Who the FBI detained $t$ ] have been released for lack of evidence.
a. who-TFR: It was reported that [TFR who officers deem $t$ to be [sc $t$ illegal immigrants]] are being separated from their children.
b. who-SFR: It was reported that [SFR who officers handcuffed $t]$ were being separated from their children.

In sum: who-TFRs exist, parallel to what-TFRs, and exhibit two characteristic properties of TFRs as opposed to SFRs in allowing indefinite interpretations and plural agreement.

\footnotetext{
${ }^{1}$ While the rating for $(11 \mathrm{~b})$ is not as high as our intuitions led us to expect, it is much higher than the ungrammatical examples in (7).
} 
5. Parallel degradation in who-TFRs and what-TFRs. The existence of who-TFRs may have been overlooked because they suffer from an ill-understood degradation in acceptability shared with many who-SFRs in English. Although there have been claims that who-FRs are always ungrammatical (Jespersen 1927:62; Bresnan \& Grimshaw 1978:340), it has been possible to find exemplars that are rated very highly (14).

(14) a. Looking through the mug shots, he suddenly proclaimed,

"That's [SFR who $t$ broke into my house]!" 6.48

b. I didn't bother with interviews, I just hired [SFR who you told me to hire $t$ ]. $\quad 6.36$

Patterson \& Caponigro (2015) (P\&C) sought to understand why good-sounding who-FRs are rare. In a rating experiment, they found that degradation in who-SFRs depends on the position of the trace inside the FR and the position of the FR in the containing clause. They tested paradigms like (15) and found that (a), where the who-FR is the matrix object and the trace of who is the object of dated, was rated much more acceptable than $(\mathrm{b}-\mathrm{c})$, which were in turn rated better than (d). ${ }^{2}$ Using their materials, we replicated this pattern in our experiment, as the ratings in (15) indicate. (The pairwise comparison (a) vs. (b) was significant $(p<.01)$, while (b) vs. (d) was marginal $(p<.08)$.)

(15) a. The music teacher married [SFR who he dated $t_{\mathrm{ACC}}$ at college]. 4.67

b. The music teacher married [SFR who $t_{\mathrm{NOM}}$ dated him at college].

c. [SFR Who the young woman met $t_{\mathrm{ACC}}$ at the party] kissed her on the way home. 2.47

d. [SFR Who $t_{\mathrm{NOM}}$ met the young woman at the party] kissed her on the way home. 2.07

If TFRs are minor variations on SFRs, then our proposed who-TFRs could be expected to show the same pattern as P\&C's who-SFRs. While the base trace of who in a TFR is always a SC subject, the structure thereabove can render its chain more subject- or object-like, allowing construction of paradigms like (16) for TFRs that are largely parallel to the SFRs in (15). In particular, the case features of the traces are the same. As expected on a unified analysis, (16) did indeed pattern like (15) in our experiment. (The following pairwise comparisons were significant: (a) vs. (b) $(p<.01)$, (c) vs. (d) $(p<.05)$, and (b) vs. (d) $(p<.01)$.) As displayed in Figure 1, where the condition letters correspond to lettered examples in (15) (light bars) and (16) (dark bars), the acceptability of who-TFRs degrades following the very same pattern as whoSFRs.

(16) a. Julie can just make out [TFR who she believes $t_{\mathrm{ACC}}$ to be [sC $t$ a security guard]] in the truck.

b. Julie can just make out [TFR who $t_{\mathrm{NOM}}$ appears to be [SC $t$ a security guard]] in the truck.

c. [TFR Who I believe $t_{\mathrm{ACC}}$ to be [SC $t$ a security guard]] is just visible in this photo. 4.80

d. [TFR Who $t_{\mathrm{NOM}}$ appears to be [SC $t$ a security guard]] is just visible in this photo. $\quad 3.87$

\footnotetext{
${ }^{2}$ What-FRs, by contrast, did not show this pattern, and were all rated more acceptable than even the best who-FRs (e.g., (15a)), in their study.
} 


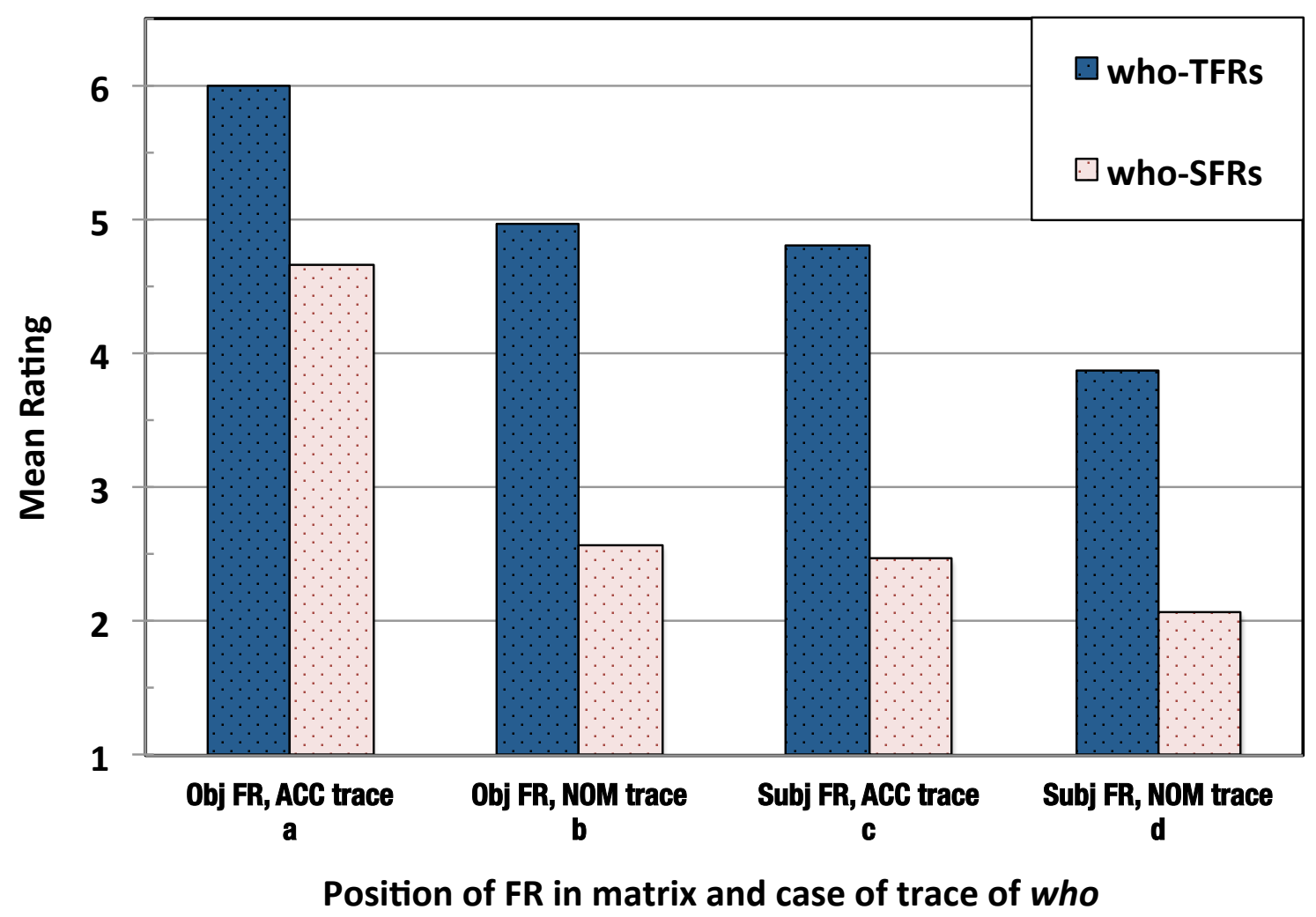

Figure 1. Mean rating for who-TFRs vs. who-SFRs according to the position of the FR in the matrix clause, and the case of the trace of who

There is additionally a main effect by which who-SFRs received lower overall ratings than who-TFRs. This may be because who-SFRs are generally degraded in (episodic) past tense contexts, as opposed to present tense ones (P\&C). P\&C chose past tense deliberately for their experimental materials, to minimize the chances of a whoever reading arising. ${ }^{3}$ On the other hand, we chose present tense deliberately in (16) to facilitate indefinite construals of who-TFRs. In any event, the main effect does not bear on our point, which is that who-TFRs show the same pattern of position-dependent degradation as who-SFRs, which is most naturally explained if they are analyzed as very similar structures.

6. Conclusion. We have provided novel data that show two ways in which TFRs parallel SFRs. For one, contrary to the view in the literature that TFRs can only be formed with what, whoTFRs exist in parallel to what-TFRs, and exhibit the characteristic behavior of TFRs in terms of indefinite interpretations and plural agreement. In addition, who-TFRs distribute in a parallel way to who-SFRs, degrading according to a position-dependent pattern. ${ }^{4}$

We take these parallels as support for analyzing TFRs as (minor variants of) SFRs (Grosu 2003, 2016), as opposed to something radically different (van Riemsdijk 2006; Wilder 1999; cf. Schelfout et al. 2004). Regarding the parallel existence of who- and what-TFRs: if TFRs are not dissimilar from SFRs, it is expected that more than one of the $w h$-words that can introduce SFRs

\footnotetext{
${ }^{3}$ Whoever-FRs show none of the degradations that who-FRs show.

${ }^{4} \mathrm{P} \& \mathrm{C}$ are unable to identify an explanation for this pattern.
} 
can also introduce TFRs. Regarding the parallel degradation of who-TFRs and who-SFRs: if TFRs are not dissimilar from SFRs, it is expected that the acceptability of who-TFRs patterns the same way as who-SFRs. From the perspective of analyses that treat TFRs radically differently from SFRs, on the other hand, these parallels of existence and degradation would be entirely coincidental.

A number of questions remain for future research. For one, do who-TFRs exist in other languages? Who-TFRs have not yet been identified in other languages that have TFRs and whoSFRs, such as German and Romanian. A possible reason for this is that whereas Wilder (1999) presented the existence of who-TFRs as an open empirical question, subsequent literature has taken the restriction to (translations of) what to be definitional of TFRs. More generally: under what circumstances do who-FRs sound best? Preliminary investigations have identified potentially relevant factors, exemplified in (14): being in a copular construction (a), or a parallel configuration that would allow verb phrase ellipsis (b).

\section{References}

Bresnan, Joan \& Jane Grimshaw. 1978. The syntax of free relatives in English. Linguistic Inquiry 9. 331-391.

Hinterwimmer, Stefan. 2008. Why free relatives sometimes behave as indefinites. In Tova Friedman \& Satoshi Ito (eds.), Proceedings of SALT XVIII. 411-428.

https://doi.org/10.3765/salt.v18i0.2514.

Grosu, Alexander. 2003. A unified theory of 'standard' and 'transparent' free relatives. Natural Language \& Linguistic Theory 21. 247-331. https://doi.org/10.1023/A:102338712.

Grosu, Alexander. 2016. The semantics, syntax and morphology of transparent free relatives revisited: A comparison of two approaches. Natural Language \&Linguistic Theory 34. 1245-1280. https://doi.org/10.1007/s11049-016-9333-0.

Jespersen, Otto. 1927. A modern English grammar on historical principles. Part III: Syntax (2nd vol.). London: George Allen \& Unwin.

Patterson, Gary \& Ivano Caponigro. 2015. The puzzling degraded status of who free relative clauses in English. English Language and Linguistics 20. 341-352.

Riemsdijk, Henk C. van. 2006. Grafts follow from Merge. In Mara Frascarelli (ed.), Phases of interpretation, 17-44. Berlin: Mouton de Gruyter.

Riemsdijk, Henk C. van. 2017. Free relatives. In Martin Everaert \& Henk C. van Riemsdijk (eds.), The Wiley Blackwell companion to syntax, second edition. Hoboken, NJ: John Wiley $\&$ Sons.

Schelfhout, Carla, Peter-Arno Coppen \& Nelleke Oostdijk. 2004. Transparent free relatives. In Sylvia Blaho, Luis Vicente, and Mark de Vos (eds.), Conference of the Student Organization of Linguistics in Europe (ConSOLE) XII, 2003, Patras. Published on-line, ISSN:1574-499X.

Wilder, Chris. 1999. Transparent free relatives. In Kimary Shahin, Susan Blake \& Eun-Sook Kim (eds.), Proceedings of the Seventeenth West Coast Conference on Formal Linguistics, 685-699. Stanford: CSLI. 